\title{
UNUSUAL POLES OF THE $\zeta$-FUNCTIONS FOR SOME REGULAR SINGULAR DIFFERENTIAL OPERATORS
}

\author{
H. FALOMIR ${ }^{A}$, M. A. MUSCHIETTI ${ }^{B}$, P. A. G. PISANI ${ }^{C}$ AND R. SEELEY ${ }^{D}$
}

\begin{abstract}
We consider the resolvent of a system of first order differential operators with a regular singularity, admitting a family of self-adjoint extensions. We find that the asymptotic expansion for the resolvent in the general case presents powers of $\lambda$ which depend on the singularity, and can take even irrational values. The consequences for the pole structure of the corresponding $\zeta$ and $\eta$-functions are also discussed.
\end{abstract}

PACS numbers: 02.30.Tb, 02.30.Sa, 03.65.Db

Mathematical Subject Classification: 81Q10, 34L05, 34L40

\section{INTRODUCTION}

It is well known that in Quantum Field Theory under external conditions, quantities like vacuum energies and effective actions, which describe the influence of boundaries or external fields on the physical system, are generically divergent and require a renormalization to get a physical meaning.

In this context, a powerful and elegant regularization scheme to deal with these problems is based on the use of the $\zeta$-function [1, 2] or the heat-kernel (for recent reviews see, for example, 3, 4, 5, 6, 7) associated to the relevant differential operators appearing in the quadratic part of the actions. In this way, ground state energies, heat-kernel coefficients, functional determinants and partition functions for quantum fields can be given in terms of the corresponding $\zeta$-function, where the ultraviolet divergent pieces of the one-loop contributions are encoded as poles of its holomorphic extension.

Thus, it is of major interest in Physics to determine the singularity structure of $\zeta$-functions associated with these physical models.

In particular [8], for an elliptic boundary value problem in a $\nu$ dimensional compact manifold with boundary, described by a differential operator $A$ of order $\omega$, with smooth coefficients and a ray of minimal growth, defined on a domain of functions subject to local boundary

Date: August 12, 2003. 
conditions, the $\zeta$-function

$$
\zeta_{A}(s) \equiv \operatorname{Tr}\left\{A^{-s}\right\}
$$

has a meromorphic extension to the complex $s$-plane whose singularities are isolated simple poles at $s=(\nu-j) / \omega$, with $j=0,1,2, \ldots$

In the case of positive definite operators, the $\zeta$-function is related, via Mellin transform, to the trace of the heat-kernel of the problem, and the pole structure of $\zeta_{A}(s)$ determines the small- $t$ asymptotic expansion of this trace 8, 9]:

$$
\operatorname{Tr}\left\{e^{-t A}\right\} \sim \sum_{j=0}^{\infty} a_{j}(A) t^{(j-\nu) / \omega},
$$

where the coefficients are related to the residues by

$$
a_{j}(A)=\left.\operatorname{Res}\right|_{s=(\nu-j) / \omega} \Gamma(s) \zeta_{A}(s) .
$$

For operators $-(d / d x)^{2}+V(x)$ with a singular potential $V(x)$ asymptotic to $g / x^{2}$ as $x \rightarrow 0$, this expansion is substantially different. If $g \geq 3 / 4$, the operator is essentially self-adjoint. This case has been treated in 10, 11, 12, where log terms are found, as well as terms with coefficients which are distributions concentrated at the singular point $x=0$. For the case $g>-1 / 4$, the Friedrichs extension has been treated in [13] for operators in $\mathbf{L}_{\mathbf{2}}(0,1)$, and in [14] for operators in $\mathbf{L}_{2}\left(\mathbf{R}^{+}\right)$.

On the other hand, reference 15 gave the pole structure of the $\zeta$ function of a second order differential operator defined on the (non compact) half-line $\mathbf{R}^{+}$, having a singular zero-th order term given by $V(x)=g x^{-2}+x^{2}$. It showed that, for a certain range of real values of $g$, this operator admits nontrivial self-adjoint extensions in $\mathbf{L}_{\mathbf{2}}\left(\mathbf{R}^{+}\right)$, for which the associated $\zeta$-function presents isolated simple poles which (in general) do not lie at $s=(1-j) / 2$ for $j=0,1, \ldots$, and can even take irrational values.

A similar structure has been noticed in [16] for the singularities of the $\zeta$-function of a system of first order differential operators in the half line, appearing in a model of Supersymmetric Quantum Mechanics with a singular superpotential $\sim x^{-1}$.

Let us mention that singular potentials $\sim 1 / x^{2}$ have been considered in the description of several physical systems, like the Calogero Model [17, 18, 15, 19, conformal invariant quantum mechanical models 20, 21, 22, and, more recently, the dynamics of quantum particles in the 
asymptotic near-horizon region of black-holes [23, 24, 25, 26, 27]. Moreover, singular superpotentials has been considered as possible agents of supersymmetry breaking in models of Supersymmetric Quantum Mechanics [28, 29, 30] (see also [16]).

It is the aim of the present article to analyze the behavior of the resolvent and $\zeta$ and $\eta$-functions of a system of first order differential operators with a regular singularity in a compact segment, admitting a family of self-adjoint extensions.

We will show that the asymptotic expansion for the resolvent in the general case presents powers of $\lambda$ which depend on the singularity, and can take even irrational values. The consequence of this behavior on the corresponding $\zeta$ and $\eta$-functions is the presence of simple poles lying at points which also depend on the singularity, with residues depending on the self-adjoint extension considered.

We first construct the resolvents for two particular extensions, for which the boundary condition at the singular point $x=0$ is invariant under the scaling $x \rightarrow c x$. The resolvent expansion for these special extensions displays the usual powers, leading to the usual poles for the $\zeta$-function. The resolvents of the remaining extensions are convex linear combinations of these special extensions, but the coefficients in the convex combination depend on the eigenvalue parameter $\lambda$. This dependence leads to unusual powers in the resolvent expansion, and hence to unusual poles for the zeta-function. These self-adjoint extensions are not invariant under the scaling $x \rightarrow c x$; as $c \rightarrow 0$ they tend (at least formally) to one of the invariant extensions, and as $c \rightarrow \infty$ they tend to the other. As $c \rightarrow 0$ the residues at the anomalous poles tend to zero, whereas as $c \rightarrow \infty$ these residues become infinite. The way these residues depend on the boundary condition is explained by a scaling argument in Section 7.

The structure of the article is as follows: In Section 2 we define the operator and determine its self-adjoint extensions, and in Section 3 we study their spectra. In Section 4 we construct the resolvent for a general extension as a linear combination of the resolvent of two limiting cases, and in Section [5 we consider the traces of these operators. The asymptotic expansion of these traces, evaluated in Section 6, is used in Section 7 to construct the associated $\zeta$ and $\eta$-functions and study their singularities.

Finally, in Section 8 we briefly describe similar results one can obtain for a second order differential operator with a regular singularity, also admitting a family of self-adjoint extensions. 


\section{The OPERATOR AND ITS SELF-ADJOINT EXTENSIONS}

Let us consider the differential operator

$$
D_{x}=\left(\begin{array}{cc}
0 & \tilde{A}_{x} \\
A_{x} & 0
\end{array}\right)
$$

with

$$
A_{x}=-\partial_{x}+\frac{g}{x}=-x^{g} \partial_{x} x^{-g}, \quad \tilde{A}_{x}=\partial_{x}+\frac{g}{x}=x^{-g} \partial_{x} x^{g},
$$

and $g \in \mathbb{R}$, defined on a domain of (two component) smooth functions with compact support in a segment, $\mathcal{D}\left(D_{x}\right)=\mathcal{C}_{0}^{\infty}(0,1)$. It can be easily seen that $D_{x}$ so defined is symmetric.

The adjoint operator $D_{x}^{*}$, which is the maximal extension of $D_{x}$, is defined on the domain $\mathcal{D}\left(D_{x}^{*}\right)$ of functions $\Phi(x)=\left(\begin{array}{c}\phi_{1}(x) \\ \phi_{2}(x)\end{array}\right) \in$ $\mathbf{L}_{\mathbf{2}}(0,1)$, having a locally summable first derivative and such that

$$
D_{x} \Phi(x)=\left(\begin{array}{c}
\tilde{A}_{x} \phi_{2}(x) \\
A_{x} \phi_{1}(x)
\end{array}\right)=\left(\begin{array}{c}
f_{1}(x) \\
f_{2}(x)
\end{array}\right) \in \mathbf{L}_{2}(0,1) .
$$

Lemma 2.1. If $\Phi(x) \in \mathcal{D}\left(D_{x}^{*}\right)$ and $-\frac{1}{2}<g<\frac{1}{2}$, then

$$
\left|\phi_{1}(x)-C_{1}[\Phi] x^{g}\right|+\left|\phi_{2}(x)-C_{2}[\Phi] x^{-g}\right| \leq K_{g}\left\|D_{x} \Phi(x)\right\| x^{1 / 2},
$$

for some constants $C_{1}[\Phi]$ and $C_{2}[\Phi]$, where $\|\cdot\|$ is the $\mathbf{L}_{\mathbf{2}}$-norm.

Indeed, Eqs. (2.3) and (2.2) imply

$$
\begin{aligned}
& \phi_{1}(x)=C_{1}[\Phi] x^{g}-x^{g} \int_{0}^{x} y^{-g} f_{2}(y) d y, \\
& \phi_{2}(x)=C_{2}[\Phi] x^{-g}+x^{-g} \int_{0}^{x} y^{g} f_{1}(y) d y,
\end{aligned}
$$

where $C_{1}[\Phi]$ and $C_{2}[\Phi]$ are integration constants which depend on the function $\Phi(x)$. Taking into account that

$$
\begin{gathered}
\left|\int_{0}^{x} y^{g} f_{1}(y) d y\right| \leq \frac{x^{g+1 / 2}}{\sqrt{1+2 g}}\left\|f_{1}\right\|, \\
\left|\int_{0}^{x} y^{-g} f_{2}(y) d y\right| \leq \frac{x^{-g+1 / 2}}{\sqrt{1-2 g}}\left\|f_{2}\right\|,
\end{gathered}
$$

we immediately get Eq. (2.4) with $K_{g}=(1-2 g)^{-1 / 2}+(1+2 g)^{-1 / 2}$. 
Lemma 2.2. Let $\Phi(x)=\left(\begin{array}{l}\phi_{1}(x) \\ \phi_{2}(x)\end{array}\right), \Psi(x)=\left(\begin{array}{l}\psi_{1}(x) \\ \psi_{2}(x)\end{array}\right) \in \mathcal{D}\left(D^{*}\right)$.

Then

$$
\begin{gathered}
\left(D_{x} \Psi, \Phi\right)-\left(\Psi, D_{x} \Phi\right)= \\
=\left\{C_{1}[\Psi]^{*} C_{2}[\Phi]-C_{2}[\Psi]^{*} C_{1}[\Phi]\right\}+\left\{\psi_{2}(1)^{*} \phi_{1}(1)-\psi_{1}(1)^{*} \phi_{2}(1)\right\} .
\end{gathered}
$$

In fact, from Eq. (2.2) one easily obtains

$$
\begin{gathered}
\left(D_{x} \Psi, \Phi\right)-\left(\Psi, D_{x} \Phi\right)= \\
=\lim _{\varepsilon \rightarrow 0^{+}} \int_{\varepsilon}^{1} \partial_{x}\left\{x^{g} \psi_{2}(x)^{*} x^{-g} \phi_{1}(x)-x^{-g} \psi_{1}(x)^{*} x^{g} \phi_{2}(x)\right\} d x,
\end{gathered}
$$

from which, taking into account the results in Lemma 2.1, Eq. (2.7) follows directly.

Now, if $\Psi(x)$ in Eq. (2.7) belongs to the domain of the closure of $D_{x}$, $\bar{D}_{x}=\left(D_{x}^{*}\right)^{*}$,

$$
\Psi(x) \in \mathcal{D}\left(\bar{D}_{x}\right) \subset \mathcal{D}\left(D_{x}^{*}\right),
$$

then the right hand side of Eq. (2.7) must vanish for any $\Phi(x) \in \mathcal{D}\left(D_{x}^{*}\right)$. Therefore

$$
C_{1}[\Psi]=0=C_{2}[\Psi] \text {, and } \Psi(1)=0
$$

On the other hand, if $\Psi(x), \Phi(x)$ belong to the domain of a symmetric extension of $D_{x}$ (contained in $\mathcal{D}\left(D_{x}^{*}\right)$ ), the right hand side of Eq. (2.7) must also vanish.

Thus, the closed extensions of $D_{x}$ correspond to the subspaces of $\mathbb{C}^{4}$ under the map $\Phi \rightarrow\left(C_{1}[\Phi], C_{2}[\Phi], \phi_{1}(1), \phi_{2}(1)\right)$, and the self-adjoint extensions correspond to those subspaces $S \subset \mathbb{C}^{4}$ such that $S=S^{\perp}$, with the orthogonal complement taken in the sense of the symplectic form on the right hand side of Eq. (2.7).

For definiteness, in the following we will consider self-adjoint extensions satisfying the local boundary condition

$$
\phi_{1}(1)=0 \text {. }
$$

Each such extension is determined by a condition of the form

$$
\alpha C_{1}[\Phi]+\beta C_{2}[\Phi]=0,
$$

with $\alpha, \beta \in \mathbb{R}$, and $\alpha^{2}+\beta^{2} \neq 0$. We denote this extension by $D_{x}^{(\alpha, \beta)}$. 


\section{THE SPECTRUM}

In order to determine the spectrum of the self-adjoint extensions of $D_{x}$, we need the solutions of

$$
\left(D_{x}-\lambda\right) \Phi(x)=0 \Rightarrow\left\{\begin{array}{l}
\tilde{A}_{x} \phi_{2}(x)=\lambda \phi_{1}(x), \\
A_{x} \phi_{1}(x)=\lambda \phi_{2}(x),
\end{array}\right.
$$

satisfying the boundary conditions in Eqs. (2.11) and (2.12).

The solution of the homogeneous equation for $\lambda=0$ is

$$
\Phi(x)=\left(\begin{array}{c}
C_{1} x^{g} \\
C_{2} x^{-g}
\end{array}\right),
$$

but the boundary conditions in Eqs. (2.11) and (2.12) imply that $C_{1}=$ 0 and $C_{2}=0$, unless $\beta=0$. Consequently, there are no zero modes except for the self-adjoint extension characterized by $\beta=0, D_{x}^{(1,0)}$.

Applying $\tilde{A}$ to the second line in Eq. (3.1), and using the first one, one easily gets

$$
\left\{\partial_{x}^{2}-\frac{g(g-1)}{x^{2}}+\lambda^{2}\right\} \phi_{1}(x)=0 .
$$

Then, for $\lambda \neq 0$, the solutions are of the form

$$
\phi_{1}(x)=K_{1} \sqrt{X} J_{\frac{1}{2}-g}(X)+K_{2} \sqrt{X} J_{g-\frac{1}{2}}(X),
$$

with $X=\tilde{\lambda} x$, where $\tilde{\lambda}=+\sqrt{\lambda^{2}}$ and $K_{1}, K_{2}$ are constants.

This implies for the lower component of $\Phi(x)$

$$
\phi_{2}(x)=\sigma\left\{-K_{1} \sqrt{X} J_{-g-\frac{1}{2}}(X)+K_{2} \sqrt{X} J_{g+\frac{1}{2}}(X)\right\},
$$

where $\sigma=\tilde{\lambda} / \lambda$.

Taking into account that

$$
J_{\nu}(X)=X^{\nu}\left\{\frac{1}{2^{\nu} \Gamma(1+\nu)}+O\left(X^{2}\right)\right\},
$$

we get

$$
\begin{gathered}
\alpha C_{1}[\Phi]+\beta C_{2}[\Phi]= \\
=\frac{\alpha K_{2} \tilde{\lambda}^{g}}{2^{g-\frac{1}{2}} \Gamma\left(\frac{1}{2}+g\right)}-\sigma \frac{\beta K_{1} \tilde{\lambda}^{-g}}{2^{-g-\frac{1}{2}} \Gamma\left(\frac{1}{2}-g\right)}=0 .
\end{gathered}
$$


For $\alpha=0$, Eq. (3.7) implies $K_{1}=0$. Therefore, $\phi_{1}(1)=0 \Rightarrow$ $J_{g-\frac{1}{2}}(\tilde{\lambda})=0$. Thus, the spectrum of this extension, $D_{x}^{(0,1)}$, is nondegenerate and symmetric with respect to the origin, with the eigenvalues given by

$$
\lambda_{ \pm, n}= \pm j_{g-\frac{1}{2}, n}, \quad n=1,2, \ldots,
$$

where $j_{\nu, n}$ is the $n$-th positive zero of the Bessel function $J_{\nu}(z)^{1}$.

For $\alpha \neq 0$, from Eq. (3.7) we can write

$$
\frac{K_{2}}{K_{1}}=\sigma \tilde{\lambda}^{-2 g}\left[\frac{4^{g} \Gamma\left(\frac{1}{2}+g\right)}{\Gamma\left(\frac{1}{2}-g\right)}\right]\left(\frac{\beta}{\alpha}\right) .
$$

In this case, the boundary condition at $x=1$ determines the eigenvalues as the solutions of the transcendental equation

$$
\tilde{\lambda}^{2 g} \frac{J_{\frac{1}{2}-g}(\tilde{\lambda})}{J_{g-\frac{1}{2}}(\tilde{\lambda})}=\sigma \rho(\alpha, \beta),
$$

where we have defined

$$
\rho(\alpha, \beta):=-\frac{4^{g} \Gamma\left(\frac{1}{2}+g\right)}{\Gamma\left(\frac{1}{2}-g\right)}\left(\frac{\beta}{\alpha}\right) .
$$

For the positive eigenvalues $\tilde{\lambda}=\lambda \Rightarrow \sigma=1$, and Eq. (3.11) reduces to

$$
F(\lambda):=\lambda^{2 g} \frac{J_{\frac{1}{2}-g}(\lambda)}{J_{g-\frac{1}{2}}(\lambda)}=\rho(\alpha, \beta),
$$

relation plotted in Figure 1 for particular values of $\rho(\alpha, \beta)$ and $g$.

On the other hand, for negative eigenvalues $\lambda=e^{i \pi} \tilde{\lambda} \Rightarrow \sigma=\tilde{\lambda} / \lambda=$ $e^{-i \pi}$, and Eq. (3.11) reads as

$$
F(\tilde{\lambda})=e^{-i \pi} \rho(\alpha, \beta)=\rho(\alpha,-\beta) .
$$

Therefore, the negative eigenvalues of $D_{x}^{(\alpha, \beta)}$ are $e^{i \pi}$ times the positive eigenvalues of $D_{x}^{(\alpha,-\beta)}$.

Notice that the spectrum is always non-degenerate, and there is a positive eigenvalue between each pair of consecutive zeroes of $J_{g-\frac{1}{2}}(\lambda)$.

${ }^{1}$ Let us recall that large zeros of $J_{\nu}(\lambda)$ have the asymptotic expansion

$$
j_{\nu, n} \simeq \gamma-\frac{4 \nu^{2}-1}{8 \gamma}+O\left(\frac{1}{\gamma}\right)^{3},
$$

with $\gamma=\left(n+\frac{\nu}{2}-\frac{1}{4}\right) \pi$. 


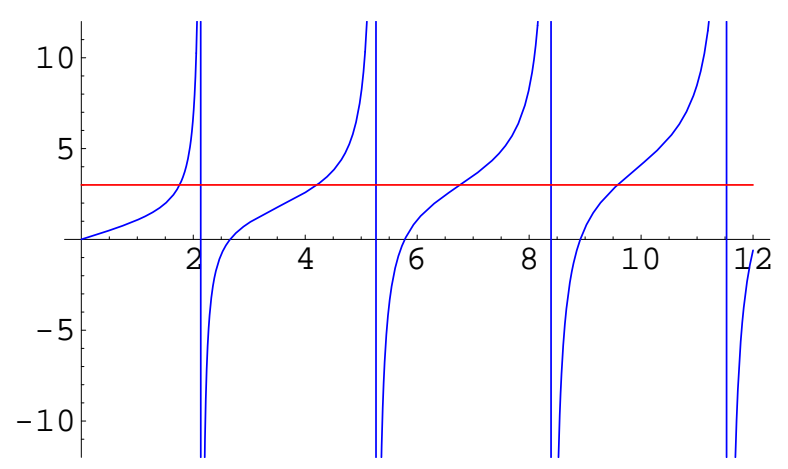

Figure 1. Plot for $F(\lambda):=\lambda^{2 g} \frac{J_{\frac{1}{2}-g}(\lambda)}{J_{g-\frac{1}{2}}(\lambda)}$, with $g=1 / 3$, and $\rho(\alpha, \beta)=3$.

Moreover, the spectrum is symmetric with respect to the origin only for the $\alpha=0$ extension (which we call the "D-extension", see Eq. (3.8)), and for the $\beta=0$ extension (which we call the "N-extension"). Indeed, in this last case, from Eqs. (3.11) and (3.12) one can see that the eigenvalues of $D_{x}^{(1,0)}$ are given by

$$
\lambda_{0}=0, \quad \lambda_{ \pm, n}= \pm j_{\frac{1}{2}-g, n}, n=1,2, \ldots
$$

\section{The RESOLVENT}

In this Section we will construct the resolvent of $D_{x}$,

$$
G(\lambda)=\left(D_{x}-\lambda\right)^{-1},
$$

for its different self-adjoint extensions.

We will first consider the two limiting cases in Eq. (2.12), namely the "D-extension", for which $\alpha=0 \Rightarrow C_{2}[\Phi]=0$, and the " $N$-extension", with $\beta=0 \Rightarrow C_{1}[\Phi]=0$. The resolvent for a general self-adjoint extension will be later evaluated as a linear combination of those obtained for these two limiting cases.

For the kernel of the resolvent

$$
G(x, y ; \lambda)=\left(\begin{array}{ll}
G_{11}(x, y ; \lambda) & G_{12}(x, y ; \lambda) \\
G_{21}(x, y ; \lambda) & G_{22}(x, y ; \lambda)
\end{array}\right)
$$


we have

$$
\left(D_{x}-\lambda\right) G(x, y ; \lambda)=\delta(x, y) \mathbf{1}_{2},
$$

from which we straightforwardly get for the diagonal elements

$$
\begin{aligned}
& \left\{\partial_{x}^{2}-\frac{g(g-1)}{x^{2}}+\lambda^{2}\right\} G_{11}(x, y ; \lambda)=-\lambda \delta(x, y), \\
& \left\{\partial_{x}^{2}-\frac{g(g+1)}{x^{2}}+\lambda^{2}\right\} G_{22}(x, y ; \lambda)=-\lambda \delta(x, y),
\end{aligned}
$$

while for the non diagonal ones we have

$$
\begin{gathered}
G_{21}(x, y ; \lambda)=\frac{1}{\lambda}\left\{-\partial_{x}+\frac{g}{x}\right\} G_{11}(x, y ; \lambda), \\
G_{12}(x, y ; \lambda)=\frac{1}{\lambda}\left\{\partial_{x}+\frac{g}{x}\right\} G_{22}(x, y ; \lambda),
\end{gathered}
$$

for $\lambda \neq 0$.

Since the resolvent is analytic in $\lambda$, it is sufficient to evaluate it on the open right half plane.

In so doing, we will need the upper and lower components of some particular solutions of the homogeneous equation (3.1).

Then, let us define

$$
\left\{\begin{array}{l}
L_{1}^{D}(X)=\sqrt{X} J_{g-\frac{1}{2}}(X), \\
L_{2}^{D}(X)=\sqrt{X} J_{g+\frac{1}{2}}(X), \\
L_{1}^{N}(X)=\sqrt{X} J_{\frac{1}{2}-g}(X), \\
L_{2}^{N}(X)=\sqrt{X} J_{-g-\frac{1}{2}}(X), \\
R_{1}(X ; \lambda)=\sqrt{X}\left[J_{g-\frac{1}{2}}(\lambda) J_{\frac{1}{2}-g}(X)-J_{\frac{1}{2}-g}(\lambda) J_{g-\frac{1}{2}}(X)\right], \\
R_{2}(X ; \lambda)=\sqrt{X}\left[J_{g-\frac{1}{2}}(\lambda) J_{-g-\frac{1}{2}}(X)+J_{\frac{1}{2}-g}(\lambda) J_{g+\frac{1}{2}}(X)\right] .
\end{array}\right.
$$

Notice that $R_{1}(\lambda ; \lambda)=0$, and $\left.\tilde{A}_{x} R_{2}(\lambda x ; \lambda)\right|_{x=1}=0$. 
We will also need the Wronskians

$$
\left\{\begin{array}{l}
W\left[L_{1}^{D}(X), R_{1}(X ; \lambda)\right]=-\frac{2}{\pi} \cos (g \pi) J_{g-\frac{1}{2}}(\lambda)=\frac{1}{\gamma_{D}(\lambda)}, \\
W\left[L_{2}^{D}(X), R_{2}(X ; \lambda)\right]=\frac{2}{\pi} \cos (g \pi) J_{g-\frac{1}{2}}(\lambda)=\frac{-1}{\gamma_{D}(\lambda)}, \\
W\left[L_{1}^{N}(X), R_{1}(X ; \lambda)\right]=-\frac{2}{\pi} \cos (g \pi) J_{\frac{1}{2}-g}(\lambda)=\frac{1}{\gamma_{N}(\lambda)}, \\
W\left[L_{2}^{N}(X), R_{2}(X ; \lambda)\right]=-\frac{2}{\pi} \cos (g \pi) J_{\frac{1}{2}-g}(\lambda)=\frac{1}{\gamma_{N}(\lambda)},
\end{array}\right.
$$

which vanish only at the zeroes of $J_{\nu}(\lambda)$, for $\nu= \pm\left(\frac{1}{2}-g\right)$.

4.1. The resolvent for the $D$-extension. In this case, the function

$$
\Phi(x)=\int_{0}^{1} G_{D}(x, y ; \lambda)\left(\begin{array}{c}
f_{1}(y) \\
f_{2}(y)
\end{array}\right) d y
$$

must satisfy $\phi_{1}(1)=0$ and $C_{2}[\Phi]=0$, for any functions $f_{1}(x), f_{2}(x) \in$ $\mathbf{L}_{\mathbf{2}}(0,1)$.

This requires that

$$
G_{11}^{D}(x, y ; \lambda)=\gamma_{D}(\lambda) \times\left\{\begin{array}{l}
L_{1}^{D}(X) R_{1}(Y ; \lambda), \text { for } x \leq y \\
R_{1}(X ; \lambda) L_{1}^{D}(Y), \text { for } x \geq y
\end{array}\right.
$$

and

$$
G_{22}^{D}(x, y ; \lambda)=-\gamma_{D}(\lambda) \times\left\{\begin{array}{l}
L_{2}^{D}(X) R_{2}(Y ; \lambda), \text { for } x \leq y, \\
R_{2}(X ; \lambda) L_{2}^{D}(Y), \text { for } x \geq y,
\end{array}\right.
$$

with the other components, $G_{12}^{D}(x, y ; \lambda)$ and $G_{21}^{D}(x, y ; \lambda)$, given as in Eq. (4.5). The fact that the boundary conditions are satisfied, as well as $\left(D_{x}-\lambda\right) \Phi(x)=\left(\begin{array}{l}f_{1}(x) \\ f_{2}(x)\end{array}\right)$, can be straightforwardly verified from Eqs. (4.6- 4.7).

Indeed, from Eqs. (4.8 - 4.10), (4.5) and (4.6 - 4.7), one gets

$$
\phi_{1}(x)=C_{1}^{D}[\Phi] x^{g}+O(\sqrt{x}), \quad \phi_{2}(x)=O(\sqrt{x}),
$$


with

$$
\begin{gathered}
C_{1}^{D}[\Phi]=\frac{-\pi \lambda^{g+1}}{2^{\frac{1}{2}+g} \cos (g \pi) J_{g-\frac{1}{2}}(\lambda) \Gamma\left(\frac{1}{2}+g\right)} \times \\
\times \int_{0}^{1}\left[R_{1}(\lambda y ; \lambda) f_{1}(y)-R_{2}(\lambda y ; \lambda) f_{2}(y)\right] d y,
\end{gathered}
$$

for $\lambda$ not a zero of $J_{g-\frac{1}{2}}(\lambda)$.

Notice that $C_{1}^{D}[\Phi] \neq 0$ if the integral in the right hand side of Eq. (4.12) is non vanishing.

4.2. The resolvent for the $N$-extension. In this case, the function

$$
\Phi(x)=\int_{0}^{1} G_{N}(x, y ; \lambda)\left(\begin{array}{c}
f_{1}(y) \\
f_{2}(y)
\end{array}\right) d y
$$

must satisfy $\phi_{1}(1)=0$ and $C_{1}[\Phi]=0$, for any functions $f_{1}(x), f_{2}(x) \in$ $\mathbf{L}_{2}(0,1)$.

This requires that

$$
G_{11}^{N}(x, y ; \lambda)=\gamma_{N}(\lambda) \times\left\{\begin{array}{l}
L_{1}^{N}(X) R_{1}(Y ; \lambda), \text { for } x \leq y \\
R_{1}(X ; \lambda) L_{1}^{N}(Y), \text { for } x \geq y
\end{array}\right.
$$

and

$$
G_{22}^{N}(x, y ; \lambda)=\gamma_{N}(\lambda) \times\left\{\begin{array}{l}
L_{2}^{N}(X) R_{2}(Y ; \lambda), \text { for } x \leq y \\
R_{2}(X ; \lambda) L_{2}^{N}(Y), \text { for } x \geq y
\end{array}\right.
$$

with the other components, $G_{12}^{N}(x, y ; \lambda)$ and $G_{21}^{N}(x, y ; \lambda)$, given as in Eq. (4.5). These boundary conditions, as well as the fact that $\left(D_{x}-\right.$ ג) $\Phi(x)=\left(\begin{array}{l}f_{1}(x) \\ f_{2}(x)\end{array}\right)$, can be straightforwardly verified from Eq. 4.6 4.7).

This time, from Eqs. (4.13- 4.15), (4.5) and (4.6- 4.7), one gets

$$
\phi_{1}(x)=O(\sqrt{x}), \quad \phi_{2}(x)=C_{2}^{N}[\Phi] x^{-g}+O(\sqrt{x}),
$$


with

$$
\begin{aligned}
& C_{2}^{N}[\Phi]=\frac{\pi \lambda^{1-g}}{2^{\frac{1}{2}-g} \cos (g \pi) J_{\frac{1}{2}-g}(\lambda) \Gamma\left(\frac{1}{2}-g\right)} \times \\
& \quad \times \int_{0}^{1}\left[R_{1}(\lambda y ; \lambda) f_{1}(y)-R_{2}(\lambda y ; \lambda) f_{2}(y)\right] d y,
\end{aligned}
$$

for $\lambda$ not a zero of $J_{\frac{1}{2}-g}(\lambda)$.

Notice that $C_{2}^{N}[\Phi] \neq 0$ if the integral in the right hand side of Eq. (4.17) (the same integral as the one appearing in the $D$-extension, Eq. (4.12) ) is non vanishing.

\subsection{The resolvent for a general self-adjoint extension of $D_{x}$.} For the general case, we can adjust the boundary conditions

$$
\phi_{1}(1)=0, \quad \alpha C_{1}[\Phi]+\beta C_{2}[\Phi]=0, \alpha, \beta \neq 0,
$$

for

$$
\Phi(x)=\int_{0}^{1} G(x, y ; \lambda)\left(\begin{array}{l}
f_{1}(y) \\
f_{2}(y)
\end{array}\right) d y,
$$

for any $f_{1}(x), f_{2}(x) \in \mathbf{L}_{\mathbf{2}}(0,1)$, by taking a linear combination of the resolvent for the limiting cases,

$$
G(x, y ; \lambda)=[1-\tau(\lambda)] G_{D}(x, y ; \lambda)+\tau(\lambda) G_{N}(x, y ; \lambda) .
$$

Since the boundary condition at $x=1$ is automatically fulfilled, one must just impose

$$
\alpha[1-\tau(\lambda)] C_{1}^{D}[\Phi]+\beta \tau(\lambda) C_{2}^{N}[\Phi]=0 .
$$

Notice that, in view of Eq. (4.12), (4.17) and (3.13),

$$
\alpha C_{1}^{D}[\Phi]-\beta C_{2}^{N}[\Phi]=0
$$

precisely when $\lambda$ is an eigenvalue of $D_{x}^{(\alpha, \beta)}$. Therefore, from Eq. (4.21) we get the resolvent of $D_{x}^{(\alpha, \beta)}$ by setting

$$
\begin{gathered}
\tau(\lambda)=\frac{\alpha C_{1}^{D}[\Phi]}{\alpha C_{1}^{D}[\Phi]-\beta C_{2}^{N}[\Phi]}=\frac{1}{1-\frac{\rho(\alpha, \beta)}{F(\lambda)}}= \\
=1-\frac{1}{1-\frac{\lambda^{2 g}}{\rho(\alpha, \beta)} \frac{J_{\frac{1}{2}-g}(\lambda)}{J_{g-\frac{1}{2}}(\lambda)}},
\end{gathered}
$$


for $\lambda$ not a zero of $J_{g-\frac{1}{2}}(\lambda)$.

\section{THE TRACE OF THE RESOLVENT}

It follows from Eq. (4.20) that the resolvent of a general self-adjoint extension of $D_{x}$ can be expressed in terms of the resolvents of the two limiting cases, $G_{D}(\lambda)$ and $G_{N}(\lambda)$. Moreover, since the eigenvalues of any extension grow linearly with $n$ (see Section [3), these resolvents are Hilbert-Schmidt operators and their $\lambda$-derivatives are trace class.

So, let us consider the relation

$$
\begin{gathered}
G^{2}(\lambda)=\partial_{\lambda} G(\lambda)=\partial_{\lambda} G_{D}(\lambda)- \\
-\tau^{\prime}(\lambda)\left[G_{D}(\lambda)-G_{N}(\lambda)\right]-\tau(\lambda)\left[\partial_{\lambda} G_{D}(\lambda)-\partial_{\lambda} G_{N}(\lambda)\right],
\end{gathered}
$$

from which it follows that the difference $G_{D}(\lambda)-G_{N}(\lambda)$ is a strongly analytic function of $\lambda$ (except at the zeroes of $\tau^{\prime}(\lambda)$ ) taking values in the trace class operators ideal.

Since we have explicitly constructed $G_{D}(\lambda)$ and $G_{N}(\lambda)$ in the previous Section (see Eqs. (4.9), (4.10), (4.14) and (4.15)), we straightforwardly get (see Appendix $\mathrm{A}$ for the details)

$$
\begin{gathered}
\operatorname{Tr}\left\{\partial_{\lambda} G_{D}(\lambda)\right\}=\int_{0}^{1} \operatorname{tr}\left\{\partial_{\lambda} G_{D}(x, x ; \lambda)\right\} d x= \\
=\partial_{\lambda}\left\{\frac{J_{g+\frac{1}{2}}(\lambda)}{J_{g-\frac{1}{2}}(\lambda)}\right\}=1-\frac{2 g}{\lambda} \frac{J_{g+\frac{1}{2}}(\lambda)}{J_{g-\frac{1}{2}}(\lambda)}+\frac{J_{g+\frac{1}{2}}^{2}(\lambda)}{J_{g-\frac{1}{2}}^{2}(\lambda)}= \\
=1-\frac{g^{2}}{\lambda^{2}}+\left(\frac{1}{2 \lambda}+\frac{J_{g-\frac{1}{2}}^{\prime}(\lambda)}{J_{g-\frac{1}{2}}(\lambda)}\right)^{2},
\end{gathered}
$$

where, in the last step, we have taken into account that

$$
J_{\nu \pm 1}(z)=\frac{\nu}{z} J_{\nu}(z) \mp J_{\nu}^{\prime}(z) .
$$

Similarly,

$$
\begin{gathered}
\operatorname{Tr}\left\{G_{D}(\lambda)-G_{N}(\lambda)\right\}=\frac{2 g}{\lambda}+\frac{J_{g+\frac{1}{2}}(\lambda)}{J_{g-\frac{1}{2}}(\lambda)}+\frac{J_{-g-\frac{1}{2}}(\lambda)}{J_{-g+\frac{1}{2}}(\lambda)}= \\
=-\frac{2 g}{\lambda}-\frac{J_{\frac{1}{2}-g}^{\prime}(\lambda)}{J_{\frac{1}{2}-g}(\lambda)}+\frac{J_{g-\frac{1}{2}}^{\prime}(\lambda)}{J_{g-\frac{1}{2}}(\lambda)} .
\end{gathered}
$$


Moreover, since

$$
\partial_{\lambda} \operatorname{Tr}\left\{G_{D}(\lambda)-G_{N}(\lambda)\right\}=\operatorname{Tr}\left\{\partial_{\lambda} G_{D}(\lambda)-\partial_{\lambda} G_{N}(\lambda)\right\}
$$

we get

$$
\begin{gathered}
\operatorname{Tr}\left\{\partial_{\lambda} G_{D}(\lambda)-\partial_{\lambda} G_{N}(\lambda)\right\}= \\
=-\frac{2 g}{\lambda^{2}}-\frac{2 g}{\lambda}\left[\frac{J_{g+\frac{1}{2}}(\lambda)}{J_{g-\frac{1}{2}}(\lambda)}+\frac{J_{-g-\frac{1}{2}}(\lambda)}{J_{-g+\frac{1}{2}}(\lambda)}\right]+\left[\frac{J_{g+\frac{1}{2}}^{2}(\lambda)}{J_{g-\frac{1}{2}}^{2}(\lambda)}-\frac{J_{-g-\frac{1}{2}}^{2}(\lambda)}{J_{-g+\frac{1}{2}}^{2}(\lambda)}\right]= \\
=\frac{2 g}{\lambda^{2}}+\left(\frac{1}{2 \lambda}+\frac{J_{\frac{1}{2}-g}^{\prime}(\lambda)}{J_{\frac{1}{2}-g}(\lambda)}\right)^{2}-\left(\frac{1}{2 \lambda}+\frac{J_{g-\frac{1}{2}}^{\prime}(\lambda)}{J_{g-\frac{1}{2}}(\lambda)}\right)^{2} .
\end{gathered}
$$

Finally, we can also write

$$
\operatorname{Tr}\left\{G^{2}(\lambda)\right\}=\operatorname{Tr}\left\{\partial_{\lambda} G_{D}(\lambda)\right\}-\partial_{\lambda}\left[\tau(\lambda) \operatorname{Tr}\left\{G_{D}(\lambda)-G_{N}(\lambda)\right\}\right]
$$

\section{Asymptotic expansion for the trace of the RESOlVEnt}

Using the Hankel asymptotic expansion for Bessel functions 32 (see Appendix $(\mathrm{B})$, we get for the first term in the right hand side of Eq. (5.7)

$$
\begin{gathered}
\operatorname{Tr}\left\{\partial_{\lambda} G_{D}(\lambda)\right\} \sim \sum_{k=2}^{\infty} \frac{A_{k}(g, \sigma)}{\lambda^{k}}= \\
=-\frac{g}{\lambda^{2}}+i \sigma \frac{g(g-1)}{\lambda^{3}}-\frac{3}{2} \frac{g(g-1)}{\lambda^{4}}+ \\
+i \sigma \frac{(g-3)(g-1) g(g+2)}{2 \lambda^{5}}+O\left(\frac{1}{\lambda}\right)^{6},
\end{gathered}
$$

where $\sigma=1$ for $\Im(\lambda)>0$, and $\sigma=-1$ for $\Im(\lambda)<0$. The coefficients in this series can be straightforwardly evaluated from Eqs. (B.7) and (B.17). Notice that $A_{k}(g,-1)=A_{k}(g, 1)^{*}$, since $A_{2 k}(g, 1)$ is real and $A_{2 k+1}(g, 1)$ is pure imaginary.

Similarly, from Eqs. (5.4), (5.6) and (B.20) we simply get

$$
\operatorname{Tr}\left\{G_{D}(\lambda)-G_{N}(\lambda)\right\} \sim-\frac{2 g}{\lambda}
$$

and

$$
\operatorname{Tr}\left\{\partial_{\lambda} G_{D}(\lambda)-\partial_{\lambda} G_{N}(\lambda)\right\} \sim \frac{2 g}{\lambda^{2}}
$$


On the other hand, taking into account Eq. (B.9), we have

$$
\begin{gathered}
\tau(\lambda) \sim 1-\frac{1}{1-\frac{e^{\sigma i \pi\left(\frac{1}{2}-g\right)} \lambda^{2 g}}{\rho(\alpha, \beta)}} \sim \\
\sim\left\{\begin{array}{l}
-\sum_{k=1}^{\infty}\left(\frac{e^{\sigma i \pi\left(\frac{1}{2}-g\right)} \lambda^{2 g}}{\rho(\alpha, \beta)}\right)^{k}, \text { for }-\frac{1}{2}<g<0, \\
\sum_{k=0}^{\infty}\left(\rho(\alpha, \beta) e^{-\sigma i \pi\left(\frac{1}{2}-g\right)} \lambda^{-2 g}\right)^{k}, \text { for } 0<g<\frac{1}{2},
\end{array}\right.
\end{gathered}
$$

where $\sigma=1(\sigma=-1)$ corresponds to $\Im(\lambda)>0(\Im(\lambda)<0)$. Notice the appearance of non integer, $g$-dependent, powers of $\lambda$ in this asymptotic expansion.

Similarly

$$
\begin{gathered}
\tau^{\prime}(\lambda) \sim-\left(1-\frac{e^{\sigma i \pi\left(\frac{1}{2}-g\right)} \lambda^{2 g}}{\rho(\alpha, \beta)}\right)^{-2} \frac{e^{\sigma i \pi\left(\frac{1}{2}-g\right)} 2 g \lambda^{2 g-1}}{\rho(\alpha, \beta)} \sim \\
\sim\left\{\begin{array}{l}
-\frac{2 g}{\lambda} \sum_{k=1}^{\infty} k\left(\frac{e^{\sigma i \pi\left(\frac{1}{2}-g\right)} \lambda^{2 g}}{\rho(\alpha, \beta)}\right)^{k}, \text { for }-\frac{1}{2}<g<0, \\
-\frac{2 g}{\lambda} \sum_{k=1}^{\infty} k\left(\rho(\alpha, \beta) e^{-\sigma i \pi\left(\frac{1}{2}-g\right)} \lambda^{-2 g}\right)^{k}, \text { for } 0<g<\frac{1}{2}
\end{array}\right.
\end{gathered}
$$

which are the term by term derivatives of the corresponding asymptotic series in Eq. 6.4).

Therefore, we have

$$
\sim\left\{\begin{array}{c}
\partial_{\lambda}\left[\tau(\lambda) \operatorname{Tr}\left\{G_{D}(\lambda)-G_{N}(\lambda)\right\}\right] \sim \\
2 g \sum_{k=1}^{\infty}\left(\frac{e^{\sigma i \pi\left(\frac{1}{2}-g\right)}}{\rho(\alpha, \beta)}\right)^{k}(2 g k-1) \lambda^{2 g k-2}, \text { for }-\frac{1}{2}<g<0, \\
2 g \sum_{k=0}^{\infty}\left(\rho(\alpha, \beta) e^{-\sigma i \pi\left(\frac{1}{2}-g\right)}\right)^{k}(2 g k+1) \lambda^{-2 g k-2}, \text { for } 0<g<\frac{1}{2} .
\end{array}\right.
$$


Notice the $g$-dependent powers of $\lambda$ appearing in these asymptotic expansions.

\section{THE $\zeta$ AND $\eta$ FUnCTIONS}

The $\zeta$-function for a general self-adjoint extension of $D_{x}$ is defined, for $\Re(s)>1$, as $[8$

$$
\zeta(s)=-\frac{1}{2 \pi i} \oint_{\mathcal{C}} \frac{\lambda^{1-s}}{s-1} \operatorname{Tr}\left\{G^{2}(\lambda)\right\} d \lambda,
$$

where the curve $\mathcal{C}$ encircles counterclockwise the spectrum of the operator, keeping to the left of the origin. According to Eq. (5.7), we have

$$
\zeta(s)=\zeta^{D}(s)+\frac{1}{2 \pi i} \oint_{\mathcal{C}} \frac{\lambda^{1-s}}{s-1} \partial_{\lambda}\left[\tau(\lambda) \operatorname{Tr}\left\{G_{D}(\lambda)-G_{N}(\lambda)\right\}\right] d \lambda
$$

where $\zeta^{D}(s)$ is the $\zeta$-function for the $D$-extension.

Since the negative eigenvalues of the self-adjoint extension of $D_{x}$ characterized by the pair $(\alpha, \beta), D_{x}^{(\alpha, \beta)}$, are minus the positive eigenvalues corresponding to the extension $D_{x}^{(\alpha,-\beta)}$ (as discussed in the Section (3), we define a partial $\zeta$-function through a path of integration encircling the positive eigenvalues only,

$$
\begin{gathered}
\zeta_{+}^{(\alpha, \beta)}(s)=\frac{1}{2 \pi i} \int_{-i \infty+0}^{i \infty+0} \frac{\lambda^{1-s}}{s-1} \operatorname{Tr}\left\{G^{2}(\lambda)\right\} d \lambda= \\
=\zeta_{+}^{D}(s)-\frac{1}{2 \pi i} \int_{-i \infty+0}^{i \infty+0} \frac{\lambda^{1-s}}{s-1} \partial_{\lambda}\left[\tau(\lambda) \operatorname{Tr}\left\{G_{D}(\lambda)-G_{N}(\lambda)\right\}\right] d \lambda,
\end{gathered}
$$

where $\zeta_{+}^{D}(s)$ is the partial $\zeta$-function for the $D$-extension.

We can also write

$$
\begin{aligned}
& \zeta_{+}^{(\alpha, \beta)}(s)=\frac{1}{2 \pi} \int_{1}^{\infty} e^{i \frac{\pi}{2}(1-s)} \frac{\mu^{1-s}}{s-1} \operatorname{Tr}\left\{G^{2}\left(e^{i \frac{\pi}{2}} \mu\right)\right\} d \mu+ \\
& +\frac{1}{2 \pi} \int_{1}^{\infty} e^{-i \frac{\pi}{2}(1-s)} \frac{\mu^{1-s}}{s-1} \operatorname{Tr}\left\{G^{2}\left(e^{-i \frac{\pi}{2}} \mu\right)\right\} d \mu+\frac{h_{1}(s)}{s-1}
\end{aligned}
$$

where $h_{1}(s)$ is an entire function. Therefore, in order to determine the poles of $\zeta_{+}^{(\alpha, \beta)}(s)$, we can subtract and add a partial sum of the asymptotic expansion obtained in the previous Section to $\operatorname{Tr}\left\{G^{2}(\lambda)\right\}$ in the integrands in the right hand side of Eq. (7.4). 
In so doing, we get for the $D$-extension and for a real $s>1$

$$
\begin{gathered}
\zeta_{+}^{D}(s)=\frac{1}{2 \pi(s-1)} \int_{1}^{\infty} e^{i \frac{\pi}{2}(1-s)} \mu^{1-s}\left\{\sum_{k=2}^{N} e^{-i \frac{\pi}{2} k} A_{k}(g, 1) \mu^{-k}\right\} d \mu+ \\
\frac{1}{2 \pi(s-1)} \int_{1}^{\infty} e^{-i \frac{\pi}{2}(1-s)} \mu^{1-s}\left\{\sum_{k=2}^{N} e^{i \frac{\pi}{2} k} A_{k}(g, 1)^{*} \mu^{-k}\right\} d \mu+\frac{h_{2}(s)}{s-1}= \\
=\frac{1}{\pi(s-1)} \sum_{k=2}^{N} \frac{1}{s-(2-k)} \Re\left\{e^{i \frac{\pi}{2}(1-s-k)} A_{k}(g, 1)\right\}+\frac{h_{2}(s)}{s-1},
\end{gathered}
$$

where $h_{2}(s)$ is an analytic function in the open half plane $\Re(s)>1-N$.

Consequently, the meromorphic extension of $\zeta_{+}^{D}(s)$ presents a simple pole at $s=1$ (see Eq. (7.3)), with a residue given by (see Eq. (5.2) )

$$
\left.\operatorname{Res} \zeta_{+}^{D}(s)\right|_{s=1}=\frac{1}{2 \pi i} \int_{-i \infty+0}^{i \infty+0} \lambda^{0} \partial_{\lambda}\left\{\frac{J_{g+\frac{1}{2}}(\lambda)}{J_{g-\frac{1}{2}}(\lambda)}\right\} d \lambda=\frac{1}{\pi}
$$

where we have used Eqs. (B.10) and (B.11).

It also presents simple poles at $s=2-k$, for $k=2,3, \ldots$, with residues given by

$$
\left.\operatorname{Res} \zeta_{+}^{D}(s)\right|_{s=2-k}=\frac{\Re\left\{i A_{k}(g, 1)\right\}}{(k-1) \pi}
$$

with the coefficients $A_{k}(g, 1)$ given in Eq. (6.1). In particular, notice that these residues vanish for even $k$.

For a general self-adjoint extension $D_{x}^{(\alpha, \beta)}$, we must also consider the singularities coming from the asymptotic expansion of $\partial_{\lambda}\left[\tau(\lambda) \operatorname{Tr}\left\{G_{D}(\lambda)-\right.\right.$ $\left.\left.G_{N}(\lambda)\right\}\right]$ in Eq. (6.6). For definiteness, let us consider in the following the case $-\frac{1}{2}<g<0$ (the case $0<g<\frac{1}{2}$ leads to similar results). 
From Eq. (7.3), and taking into account Eq. (7.4), for real $s>1$ we can write

$$
\begin{gathered}
\zeta_{+}^{(\alpha, \beta)}(s)-\zeta_{+}^{D}(s)=\frac{h_{3}(s)}{s-1}- \\
-\frac{g}{\pi(s-1)} \int_{1}^{\infty} e^{i \frac{\pi}{2}(-s-1)} \mu^{1-s}\left\{\sum_{k=1}^{N}\left(\frac{e^{i \frac{\pi}{2}}}{\rho(\alpha, \beta)}\right)^{k}(2 g k-1) \mu^{2 g k-2}\right\} d \mu \\
-\frac{g}{\pi(s-1)} \int_{1}^{\infty} e^{-i \frac{\pi}{2}(-s-1)} \mu^{1-s}\left\{\sum_{k=1}^{N}\left(\frac{e^{-i \frac{\pi}{2}}}{\rho(\alpha, \beta)}\right)^{k}(2 g k-1) \mu^{2 g k-2}\right\} d \mu \\
=\frac{-2 g}{\pi(s-1)} \sum_{k=1}^{N}\left(\frac{2 g k-1}{s-2 g k}\right) \Re\left\{\frac{e^{i \frac{\pi}{2}(k-s-1)}}{\rho(\alpha, \beta)^{k}}\right\}+\frac{h_{3}(s)}{s-1},
\end{gathered}
$$

where $h_{3}(s)$ is holomorphic for $\Re(s)>2 g(N+1)$.

Therefore, $\left(\zeta_{+}^{(\alpha, \beta)}(s)-\zeta_{+}^{D}(s)\right)$ has a meromorphic extension which presents a simple pole at $s=1$, with a vanishing residue,

$$
\begin{gathered}
\left.\operatorname{Res}\left(\zeta_{+}^{(\alpha, \beta)}(s)-\zeta_{+}^{D}(s)\right)\right|_{s=1}= \\
=-\frac{1}{2 \pi i} \int_{-i \infty+0}^{i \infty+0} \lambda^{0} \partial_{\lambda}\left[\tau(\lambda) \operatorname{Tr}\left\{G_{D}(\lambda)-G_{N}(\lambda)\right\}\right] d \lambda=0,
\end{gathered}
$$

as follows from Eqs. (6.2) and (6.4).

Notice also the presence of simple poles located at negative non integer $g$-dependent positions, $s=2 g k=-2|g| k$, for $k=1,2, \ldots$, with residues which also depend on the self-adjoint extension, given by

$$
\begin{aligned}
& \left.\operatorname{Res}\left\{\zeta_{+}^{(\alpha, \beta)}(s)-\zeta_{+}^{D}(s)\right\}\right|_{s=2 g k}= \\
& =\frac{-2 g}{\pi \rho(\alpha, \beta)^{k}} \sin \left[\left(\frac{1}{2}-g\right) k \pi\right] .
\end{aligned}
$$

Now, taking into account our comment after Eq. (3.14), we get for the complete $\zeta$-function

$$
\zeta^{(\alpha, \beta)}(s)=\zeta_{+}^{(\alpha, \beta)}(s)+e^{-i \pi s} \zeta_{+}^{(\alpha,-\beta)}(s) .
$$

In particular, for the $\alpha=0$ extension we get

$$
\zeta^{D}(s)=\left(1+e^{-i \pi s}\right) \zeta_{+}^{D}(s),
$$


since the spectrum of $D_{x}^{(0,1)}$ is symmetric with respect to the origin (see Eq. (3.8) ). Then one concludes that $\zeta^{D}(s)$ has vanishing residues. Indeed, from Eq. (7.7), the residue at $s=2-k$ vanishes for $k$ even, and for $k=2 l+1$, with $l=0,1,2, \ldots$, we have

$$
\left.\operatorname{Res}\left\{\zeta^{D}(s)\right\}\right|_{s=1-2 l}=\left.\left(1+e^{-i \pi(1-2 l)}\right) \operatorname{Res}\left\{\zeta_{+}^{D}(s)\right\}\right|_{s=1-2 l}=0 .
$$

On the other hand, for a general self-adjoint extension, the singularities of $\zeta^{(\alpha, \beta)}(s)$ are simple poles located at $s=2 g k<0$, for $k=1,2, \ldots$, with residues

$$
\begin{gathered}
\left.\operatorname{Res}\left\{\zeta^{(\alpha, \beta)}(s)-\zeta^{D}(s)\right\}\right|_{s=2 g k}= \\
=\left.\operatorname{Res}\left\{\left[\zeta_{+}^{(\alpha, \beta)}(s)-\zeta_{+}^{D}(s)\right]+e^{-i \pi s}\left[\zeta_{+}^{(\alpha,-\beta)}(s)-\zeta_{+}^{D}(s)\right]\right\}\right|_{s=2 g k}= \\
=(-1)^{k} \frac{2 g}{\pi} \frac{\sin (2 g k \pi)}{\rho(\alpha, \beta)^{k}} e^{i \pi\left(\frac{1}{2}-g\right) k}
\end{gathered}
$$

where we have used $\rho(\alpha,-\beta)=-\rho(\alpha, \beta)$, from Eq. (3.12).

Similarly, for the spectral asymmetry 31] we have

$$
\eta^{(\alpha, \beta)}(s)=\zeta_{+}^{(\alpha, \beta)}(s)-\zeta_{+}^{(\alpha,-\beta)}(s) .
$$

In particular, $\eta^{(0,1)}(s) \equiv 0 \equiv \eta^{(1,0)}(s)$, since these spectra are symmetric (see Eqs. (3.8) and (3.15) ).

For a general self-adjoint extension and $-\frac{1}{2}<g<0, \eta^{(\alpha, \beta)}(s)$ presents simple poles at $s=2 g k$, for $k=1,2, \ldots$, with residues given by

$$
\left.\operatorname{Res}\left\{\eta^{(\alpha, \beta)}(s)\right\}\right|_{s=2 g k}=\left[(-1)^{k}-1\right] \frac{2 g}{\pi} \frac{\sin \left[\left(\frac{1}{2}-g\right) k \pi\right]}{\rho(\alpha, \beta)^{k}},
$$

which vanish for even $k$.

For the case $0<g<\frac{1}{2}$, an entirely similar calculation shows that $\left(\zeta_{+}^{(\alpha, \beta)}(s)-\zeta_{+}^{D}(s)\right)$ has a meromorphic extension which presents simple poles at negative non integer $g$-dependent positions, $s=-2 g k$, for $k=1,2, \ldots$, with residues depending on the self-adjoint extension, 
given by

$$
\begin{gathered}
\left.\operatorname{Res}\left\{\zeta_{+}^{(\alpha, \beta)}(s)-\zeta_{+}^{D}(s)\right\}\right|_{s=-2 g k}= \\
=-\frac{2 g}{\pi} \rho(\alpha, \beta)^{k} \sin \left[\left(\frac{1}{2}-g\right) k \pi\right] .
\end{gathered}
$$

From this result, it is immediate to get the residues for the $\eta$ and $\zeta$ functions. One gets the same expressions as in the right hand sides of Eqs. (7.14) and (7.16), with $\rho(\alpha, \beta)$ and $e^{i \pi\left(\frac{1}{2}-g\right) k}$ replaced by their inverses.

Let us remark that when neither $\alpha$ nor $\beta$ is 0 , the residue of $\zeta_{+}^{(\alpha, \beta)}$ at $s=-2|g| k$ is a constant times $(\beta / \alpha)^{k \operatorname{sign}(g)}$. This is consistent with the behavior of $D_{x}$ under the scaling isometry $T u(x)=c^{1 / 2} u(c x)$ taking $\mathbf{L}_{\mathbf{2}}(0,1) \rightarrow \mathbf{L}_{\mathbf{2}}(0,1 / c)$. The extension $D_{x}^{(\alpha, \beta)}$ is unitarily equivalent to the operator $(1 / c) \dot{D}_{x}^{\left(\alpha^{\prime}, \beta^{\prime}\right)}$ similarly defined on $\mathbf{L}_{\mathbf{2}}(0,1 / c)$, with $\alpha^{\prime}=$ $c^{-g} \alpha$ and $\beta^{\prime}=c^{g} \beta$ :

$$
T D_{x}^{(\alpha, \beta)}=\frac{1}{c} \dot{D}_{x}^{\left(\alpha^{\prime}, \beta^{\prime}\right)} T .
$$

Notice that only for the extensions with $\alpha=0$ or $\beta=0$ the boundary condition at the singular point $x=0$, Eq. (2.12), is left invariant by this scaling.

Therefore, we have for the partial $\zeta$-function of the scaled problem

$$
\dot{\zeta}_{+}^{\left(\alpha^{\prime}, \beta^{\prime}\right)}(s)=c^{-s} \zeta_{+}^{(\alpha, \beta)}(s),
$$

and for the residues

$$
\left.\operatorname{Res}\left\{\dot{\zeta}_{+}^{\left(\alpha^{\prime}, \beta^{\prime}\right)}(s)\right\}\right|_{s=-2|g| k}=\left.c^{2|g| k} \operatorname{Res}\left\{\zeta_{+}^{(\alpha, \beta)}(s)\right\}\right|_{s=-2|g| k} .
$$

The factor $c^{2|g| k}$ exactly cancels the effect the change in the boundary condition at the singularity has on $\rho(\alpha, \beta)$,

$$
\rho(\alpha, \beta)^{k \operatorname{sign}(g)}=c^{-2|g| k} \rho\left(\alpha^{\prime}, \beta^{\prime}\right)^{k \operatorname{sign}(g)} .
$$

Thus the difference between the intervals $(0,1)$ and $(0,1 / c)$ has no effect on the structure of these residues, which presumably are determined locally in a neighborhood of $x=0$.

Finally, let us point out that these anomalous poles are not present in the $g=0$ case. Indeed, in this case $\tau(\lambda)$ in Eq. (4.23) has a constant asymptotic expansion, while $\operatorname{Tr}\left\{G_{D}(\lambda)-G_{N}(\lambda)\right\} \sim 0$ (see Eq. (6.2)). Moreover, the residues of the poles coming from $\zeta_{+}^{D}(s)$ are all zero (see 
Eqs. (7.7) and (6.1) ), except for the one at $s=1$, with residue $1 / \pi$ (see Eq. (17.6) ).

Consequently, the presence of poles in the spectral functions located at non integer positions is a consequence of the singular behavior of the 0 -th order term in $D_{x}$ near the origin, together with a boundary condition which is not invariant under scaling.

\section{Comments on the SECOND ORDER CASE}

In this Section we briefly describe similar results one can obtain for the self-adjoint extensions of the second order differential operator

$$
\Delta_{x}=-\partial_{x}^{2}+\frac{g(g-1)}{x^{2}}
$$

with $-\frac{1}{2}<g<\frac{1}{2}$, defined on a set of functions satisfying $\phi(1)=0$ and behaving as

$$
\phi(x)=C_{1} x^{g}+C_{2} x^{1-g}+O\left(x^{3 / 2}\right),
$$

where the coefficients $C_{1,2}$ are constrained as in Eq. (2.12).

It can be shown that the spectrum of the self-adjoint extension $\Delta_{x}^{(\alpha, \beta)}$ is determined by a relation similar to Eq. (3.13):

$$
\mathcal{F}(\mu):=\frac{1}{\mu} F(\mu)=\varrho(\alpha, \beta),
$$

where the constant

$$
\varrho(\alpha, \beta):=\left(\frac{\beta}{\alpha}\right) 2^{2 g-1} \frac{\Gamma\left(\frac{1}{2}+g\right)}{\Gamma\left(\frac{3}{2}-g\right)} .
$$

Also in this case, $\alpha=0$ and $\beta=0$ correspond to two scale invariant boundary conditions at the singularity. For these two limiting extensions, it is easily seen from Eqs. (4.4), (4.11) and (4.16) that the entry $G_{11}(x, y ; \mu)$ in the resolvent of our first order operator $D_{x}^{(\alpha, \beta)}$ is $\mu$ times the corresponding resolvent of $\Delta_{x}^{(\alpha, \beta)}$ at $\lambda=\mu^{2}$,

$$
\mathcal{G}_{D, N}\left(x, y ; \mu^{2}\right)=\frac{1}{\mu} G_{11}^{D, N}(x, y ; \mu) .
$$

The resolvent for a general self-adjoint extension $\Delta_{x}^{(\alpha, \beta)}$ is constructed as a convex linear combination of $\mathcal{G}_{D}\left(\mu^{2}\right)$ and $\mathcal{G}_{N}\left(\mu^{2}\right)$ as in (4.20), with a coefficient

$$
\tau(\mu)=\frac{1}{1-\frac{\varrho(\alpha, \beta)}{\mathcal{F}(\mu)}}
$$


Following the methods employed for the first order case, one can show that the $\zeta$-function associated to $\Delta_{x}^{(\alpha, \beta)}$ also displays anomalous poles located at $s=-\left(\frac{1}{2}-g\right) k$, with $k=1,2, \ldots$, which implies the presence of anomalous powers $t^{\left(\frac{1}{2}-g\right) k}$ in the heat trace small- $t$ asymptotic expansion. The residues at these poles, and the corresponding heat trace coefficients are similarly evaluated. More details on this calculation will be reported elsewhere.

Note added in proof: It has come belatedly to our attention that the article by Edith A. Mooers, "Heat kernel asymptotics on manifolds with conic singularities", J. Anal. Math. vol 78 (1999) 1-36, gives the first "unusual" term in the expansion of the Laplacian on the half line with a domain which is not scaling invariant. That article also gives a construction which in principle would give the complete expansion in the case of a manifold with isolated conic singularities, for an arbitrary self-adjoint realization of the Laplacian. For the case considered here, by contrast, the present results are simpler and more complete, as they treat the first order case and the eta invariant, and give more explicit coefficients.

\section{ACKNOWLEDGEMENTS}

HF and PAGP acknowledge support from Universidad Nacional de La Plata (grant 11/X298) and CONICET (grant 0459/98), Argentina. They also acknowledge support from CLAF-ICTP (grant 196/02).

MAM acknowledge support from Universidad Nacional de La Plata (grant 11/X228), Argentina.

\section{Appendix A. Evaluation of the traces}

In this Appendix we briefly describe the evaluation of the traces appearing in Section 5 .

We need to compute

$$
\begin{gathered}
\operatorname{Tr}\left\{G_{D}(\lambda)-G_{N}(\lambda)\right\}= \\
=\int_{0}^{1}\left[\operatorname{tr}\left\{G_{D}(x, x ; \lambda)\right\}-\operatorname{tr}\left\{G_{N}(x, x ; \lambda)\right\}\right] d x .
\end{gathered}
$$

Let us first consider the contribution of $G_{N}(\lambda)$ to this integral. From Eq. (4.14) and (4.15) we get for the matrix trace of $G_{N}(\lambda)$ on the 
diagonal

$$
\begin{gathered}
\operatorname{tr}\left\{G_{N}(x, x ; \lambda)\right\}=-\frac{\pi x \lambda \sec (g \pi)}{2 J_{\frac{1}{2}-g}(\lambda)}\left\{J_{-\frac{1}{2}-g}(x \lambda)^{2} J_{-\frac{1}{2}+g}(\lambda)+\right. \\
+J_{\frac{1}{2}-g}(x \lambda)\left[J_{\frac{1}{2}-g}(x \lambda) J_{-\frac{1}{2}+g}(\lambda)-J_{\frac{1}{2}-g}(\lambda) J_{-\frac{1}{2}+g}(x \lambda)\right]+ \\
\left.+J_{-\frac{1}{2}-g}(x \lambda) J_{\frac{1}{2}-g}(\lambda) J_{\frac{1}{2}+g}(x \lambda)\right\}
\end{gathered}
$$

an integrable expression behaving as

$$
\begin{gathered}
\operatorname{tr}\left\{G_{N}(x, x ; \lambda)\right\}= \\
=x^{-2 g}\left\{-\left(\frac{4^{g} \pi \sec (g \pi) J_{g-\frac{1}{2}}(\lambda)}{\lambda^{2 g} \Gamma\left(\frac{1}{2}-g\right)^{2} J_{\frac{1}{2}-g}(\lambda)}\right)+O(x)\right\}+O(x)
\end{gathered}
$$

near the origin.

Therefore, it is sufficient to know the primitives 33]

$$
\int x J_{\nu}^{2}(\lambda x) d x=\frac{x^{2}}{2}\left\{J_{\nu}(x \lambda)^{2}-J_{\nu-1}(x \lambda) J_{\nu+1}(x \lambda)\right\}
$$

and

$$
\begin{gathered}
\int x J_{\nu}(\lambda x) J_{-\nu}(\lambda x) d x= \\
=\frac{-\nu^{2}}{\lambda^{2} \Gamma(1-\nu) \Gamma(1+\nu)}\left[{ }_{1} F_{2}\left(\{-1 / 2\},\{-\nu, \nu\},-x^{2} \lambda^{2}\right)-1\right],
\end{gathered}
$$

where

(A.6)

$$
\begin{gathered}
{ }_{1} F_{2}\left(\{-1 / 2\},\{-\nu, \nu\},-x^{2} \lambda^{2}\right)=-\frac{\pi x^{2} \lambda^{2} \csc (\pi \nu)}{4 \nu} \times \\
\left\{J_{-1-\nu}(x \lambda) J_{-1+\nu}(x \lambda)+2 J_{-\nu}(x \lambda) J_{\nu}(x \lambda)+J_{1-\nu}(x \lambda) J_{1+\nu}(x \lambda)\right\} .
\end{gathered}
$$

These primitives, together with the relation

$$
J_{\nu-1}(z)+J_{\nu+1}(z)=\frac{2 \nu}{z} J_{\nu}(z)
$$

necessary to simplify the intermediate results, eventually lead to

$$
I_{N}(\lambda):=\int_{0}^{1} \operatorname{tr}\left\{G_{N}(x, x ; \lambda)\right\} d x=-\frac{2 g}{\lambda}-\frac{J_{-g-\frac{1}{2}}(\lambda)}{J_{-g+\frac{1}{2}}(\lambda)} .
$$


Similarly, for the matrix trace of $G_{D}(\lambda)$ on the diagonal we have

$$
\begin{gathered}
\operatorname{tr}\left\{G_{D}(x, x ; \lambda)\right\}= \\
=\frac{\pi x \lambda \sec (g \pi)}{2 J_{-\frac{1}{2}+g}(\lambda)}\left\{-J_{\frac{1}{2}-g}(x \lambda) J_{-\frac{1}{2}+g}(\lambda) J_{-\frac{1}{2}+g}(x \lambda)+\right. \\
+J_{-\frac{1}{2}-g}(x \lambda) J_{-\frac{1}{2}+g}(\lambda) J_{\frac{1}{2}+g}(x \lambda)+ \\
\left.+J_{\frac{1}{2}-g}(\lambda)\left(J_{-\frac{1}{2}+g}(x \lambda)^{2}+J_{\frac{1}{2}+g}(x \lambda)^{2}\right)\right\}
\end{gathered}
$$

which behaves as

$$
\begin{gathered}
\operatorname{tr}\left\{G_{D}(x, x ; \lambda)\right\}= \\
=x^{2 g}\left\{\frac{\pi \lambda^{2 g} \sec (g \pi) J_{\frac{1}{2}-g}(\lambda)}{4^{g} \Gamma\left(\frac{1}{2}+g\right)^{2} J_{g-\frac{1}{2}}(\lambda)}+O(x)\right\}+O(x) .
\end{gathered}
$$

The same argument as before leads to

$$
I_{D}(\lambda):=\int_{0}^{1} \operatorname{tr}\left\{G_{D}(x, x ; \lambda)\right\} d x=\frac{J_{g+\frac{1}{2}}(\lambda)}{J_{g-\frac{1}{2}}(\lambda)} .
$$

Therefore, we get

$$
\operatorname{Tr}\left\{G_{D}(\lambda)-G_{N}(\lambda)\right\}=I_{D}(\lambda)-I_{N}(\lambda),
$$

as in Eq. (5.4).

On the other hand, we have

$$
\begin{gathered}
\partial_{\lambda} \operatorname{tr}\left\{G_{D}(x, x ; \lambda)\right\}=O(x)+ \\
x^{2 g}\left\{\frac{2^{1-2 g} \lambda^{-1+2 g}\left[1+g \pi J_{\frac{1}{2}-g}(\lambda) J_{g-\frac{1}{2}}(\lambda) \sec (g \pi)\right]}{J_{g-\frac{1}{2}}(\lambda)^{2} \Gamma\left(\frac{1}{2}+g\right)^{2}}+O(x)\right\} .
\end{gathered}
$$

Then,

$$
\operatorname{Tr}\left\{\partial_{\lambda} G_{D}(\lambda)\right\}=\int_{0}^{1} \partial_{\lambda} \operatorname{tr}\left\{G_{D}(x, x ; \lambda)\right\} d x=\partial_{\lambda} I_{D}(\lambda),
$$

in agreement with Eq. (5.2). 


\section{Appendix B. The HANKel EXPANSION}

In order to develop an asymptotic expansion for the trace of the resolvent, we use the Hankel asymptotic expansion for the Bessel functions: For $|z| \rightarrow \infty$, with $\nu$ fixed and $|\arg z|<\pi$, we have 32 .

$$
J_{\nu}(z) \sim\left(\frac{2}{\pi z}\right)^{\frac{1}{2}}\{P(\nu, z) \cos \chi(\nu, z)-Q(\nu, z) \sin \chi(\nu, z)\}
$$

where

$$
\begin{gathered}
\chi(\nu, z)=z-\left(\frac{\nu}{2}+\frac{1}{4}\right) \pi \\
P(\nu, z) \sim \sum_{k=0}^{\infty} \frac{(-1)^{k} \Gamma\left(\frac{1}{2}+\nu+2 k\right)}{(2 k) ! \Gamma\left(\frac{1}{2}+\nu-2 k\right)} \frac{1}{(2 z)^{2 k}}
\end{gathered}
$$

and

$$
Q(\nu, z) \sim \sum_{k=0}^{\infty} \frac{(-1)^{k} \Gamma\left(\frac{1}{2}+\nu+2 k+1\right)}{(2 k+1) ! \Gamma\left(\frac{1}{2}+\nu-2 k-1\right)} \frac{1}{(2 z)^{2 k+1}} .
$$

Moreover, $P(-\nu, z)=P(\nu, z)$ and $Q(-\nu, z)=Q(\nu, z)$, since these functions depend only on $\nu^{2}$ (see Ref. [32], page 364).

Therefore, for $z$ in the upper open half plane,

$$
J_{\nu}(z) \sim \frac{e^{-i z} e^{i \pi\left(\frac{\nu}{2}+\frac{1}{4}\right)}}{\sqrt{2 \pi z}}\{P(\nu, z)-i Q(\nu, z)\}
$$

while for $z$ in the lower open half plane we get

$$
J_{\nu}(z) \sim \frac{e^{i z} e^{-i \pi\left(\frac{\nu}{2}+\frac{1}{4}\right)}}{\sqrt{2 \pi z}}\{P(\nu, z)+i Q(\nu, z)\} .
$$

In these equations,

$$
P(\nu, z) \pm i Q(\nu, z) \sim \sum_{k=0}^{\infty}\langle\nu, k\rangle\left(\frac{ \pm i}{2 z}\right)^{k}
$$

where the coefficients

$$
\langle\nu, k\rangle=\frac{\Gamma\left(\frac{1}{2}+\nu+k\right)}{k ! \Gamma\left(\frac{1}{2}+\nu-k\right)}=\langle-\nu, k\rangle
$$

are the Hankel symbols.

In particular, the quotient

$$
\frac{J_{\frac{1}{2}-g}(\lambda)}{J_{g-\frac{1}{2}}(\lambda)} \sim e^{ \pm i \pi\left(\frac{1}{2}-g\right)} \frac{P\left(\frac{1}{2}-g, \lambda\right) \mp i Q\left(\frac{1}{2}-g, \lambda\right)}{P\left(g-\frac{1}{2}, \lambda\right) \mp i Q\left(g-\frac{1}{2}, \lambda\right)}=e^{ \pm i \pi\left(\frac{1}{2}-g\right)},
$$


for $\Im(\lambda)>0$ and $\Im(\lambda)<0$ respectively, since $P(\nu, z)$ and $Q(\nu, z)$ are even in $\nu$.

For the quotient of two Bessel functions we have

$$
\frac{J_{\nu_{1}}(z)}{J_{\nu_{2}}(z)} \sim e^{ \pm i \frac{\pi}{2}\left(\nu_{1}-\nu_{2}\right)} \frac{P\left(\nu_{1}, z\right) \mp i Q\left(\nu_{1}, z\right)}{P\left(\nu_{2}, z\right) \mp i Q\left(\nu_{2}, z\right)},
$$

where the upper sign is valid for $\Im(\lambda)>0$, and the lower one for $\Im(\lambda)<0$. The coefficients of these asymptotic expansions can be easily obtained, to any order, from Eq. (B.7),

$$
\frac{P\left(\nu_{1}, z\right) \pm i Q\left(\nu_{1}, z\right)}{P\left(\nu_{2}, z\right) \pm i Q\left(\nu_{2}, z\right)} \sim 1+\left(\left\langle\nu_{1}, 1\right\rangle-\left\langle\nu_{2}, 1\right\rangle\right)\left(\frac{ \pm i}{2 z}\right)+O\left(\frac{1}{z^{2}}\right)
$$

Similarly, the derivative of the Bessel function has the following asymptotic expansion [32] for $|\arg z|<\pi$,

$$
J_{\nu}^{\prime}(z) \sim-\frac{2}{\sqrt{2 \pi z}}\{R(\nu, z) \sin \chi(\nu, z)+S(\nu, z) \cos \chi(\nu, z)\},
$$

where

$$
R(\nu, z) \sim \sum_{k=0}^{\infty}(-1)^{k} \frac{\nu^{2}+(2 k)^{2}-1 / 4}{\nu^{2}-(2 k-1 / 2)^{2}} \frac{\langle\nu, 2 k\rangle}{(2 z)^{2 k}}
$$

and

$$
S(\nu, z) \sim \sum_{k=0}^{\infty}(-1)^{k} \frac{\nu^{2}+(2 k+1)^{2}-1 / 4}{\nu^{2}-(2 k+1-1 / 2)^{2}} \frac{\langle\nu, 2 k+1\rangle}{(2 z)^{2 k+1}}
$$

Then,

$$
J_{\nu}^{\prime}(z) \sim \mp i \frac{e^{\mp i z} e^{ \pm i \pi\left(\frac{\nu}{2}+\frac{1}{4}\right)}}{\sqrt{2 \pi z}}\{R(\nu, z) \mp i S(\nu, z)\},
$$

where the upper sign is valid for $\Im(\lambda)>0$, and the lower one for $\Im(\lambda)<0$. We have also

$$
R(\nu, z) \pm i S(\nu, z)=P(\nu, z) \pm i Q(\nu, z)+T_{ \pm}(\nu, z),
$$

with

$$
T_{ \pm}(\nu, z) \sim \sum_{k=1}^{\infty}(2 k-1)\langle\nu, k-1\rangle\left(\frac{ \pm i}{2 z}\right)^{k} .
$$

Therefore, we get

$$
\frac{J_{\nu}^{\prime}(z)}{J_{\nu}(z)} \sim \mp i\left\{1+\frac{T_{\mp}(\nu, z)}{P(\nu, z) \mp i Q(\nu, z)}\right\},
$$


where the upper sign is valid for $\Im(\lambda)>0$, and the lower one for $\Im(\lambda)<0$. The coefficients of the asymptotic expansion in the right hand side of Eq. (B.18) can be easily obtained from Eq. (B.7) and (B.17),

$$
\frac{T_{ \pm}(\nu, z)}{P(\nu, z) \pm i Q(\nu, z)}=\left(\frac{ \pm i}{2 z}\right)+O\left(\frac{1}{z^{2}}\right)
$$

Finally, since the Hankel symbols are even in $\nu$ (see Eq. (B.8)), from Eq. (B.7), (B.17) and (B.18) we have

$$
\frac{J_{\nu}^{\prime}(z)}{J_{\nu}(z)} \sim \frac{J_{-\nu}^{\prime}(z)}{J_{-\nu}(z)} .
$$

\section{REFERENCES}

[1] J.S. Dowker and R. Critchley, Phys. Rev. D 13, 3224 (1976).

[2] S.W. Hawking, Commun. Math. Phys. 55, 133 (1977).

[3] E. Elizalde, S.D. Odintsov, A. Romeo, A.A. Bytsenko and S. Zerbini, Zeta Regularization Techniques with Applications. World Scientific, Singapore (1994).

[4] A.A. Bytsenko, G. Cognola, L. Vanzo and S. Zerbini, Phys. Rep. 266, 1 (1996).

[5] K. Kirsten. Spectral Functions in Mathematics and Physics, hep-th/0007251 (2000).

[6] M. Bordag, U. Mohideen and V.M. Mostepanenko. New Developments in the Casimir Effect, quant-ph/0106045 (2001).

[7] D. V. Vassilevich, Heat kernel expansion: User's manual, hep-th/0306138 (2003).

[8] R.T. Seeley. A. M. S. Proc. Symp. Pure Math. 10, 288 (1967). Am. Journ. Math. 91, 889 (1969). Am. Journ. Math. 91, 963 (1969).

[9] P.B. Gilkey, Invariance Theory, the Heat Equation and the Atiyah - Singer Index Theorem, CRC Press, Boca Ratón (1995).

[10] C.J. Callias, The heat equation with singular coefficients. I: Operators of the form $-d^{2} / d x^{2}+k / x^{2}$ in dimension 1, Commun. Math. Phys. 88, 357-385 (1983).

[11] C.J. Callias and G.A. Uhlmann, Singular asymptotics approach to partial differential equations with isolated singularities in the coefficients, Bull. Am. Math. Soc., New Ser. 11, 172-176 (1984).

[12] C.J. Callias, The resolvent and the heat kernel for some singular boundary problems, Commun. Partial Differ. Equations 13, No.9, 1113-1155 (1988).

[13] J. Brüning, Math. Ann. 268, 173-196 (1984).

[14] J. Brüning and R. Seeley, Adv. in Math. 58, 133-148 (1985).

[15] H. Falomir, P. A. G. Pisani and A. Wipf, Journal of Physics A: Mathematical and General $\underline{35}$, (2002) 5427.

[16] H. Falomir, P. A. G. Pisani, in preparation.

[17] F. Calogero, Jour. Math. Phys. 10, 2191 (1969); Jour. Math. Phys. 10, 2197 (1969); Jour. Math. Phys. 12, 419 (1971).

[18] M. A. Olshanetsky and A. M. Perelomov, Phys. Rep. 71, 313-400 (1981). 
[19] B. Basu-Mallick, P. K. Ghosh and K. S. Gupta, Phys. Lett. A 311, 87 (2003); Nucl. Phys. B 659, 437 (2003).

[20] V. De Alfaro, S. Fubini and G. Furlan, Nuovo Cim. A34, 569 (1976).

[21] H. E. Camblong, L. N. Epele, H. Fanchiotti and C. A. Garcia Canal, Annals Phys. 287, 14 (2001); Annals Phys. 287, 57 (2001).

[22] Sidney A. Coon, Barry R. Holstein, Anomalies in Quantum Mechanics: the $1 / r^{2}$ Potential, quant-ph/0202091 (2002).

[23] P. Claus, M. Derix, R. Kallosh, J. Kumar, P. K. Townsend and A. Van Proeyen, Phys. Rev. Lett. 81, 4553 (1998).

[24] G. W. Gibbons and P. K. Townsend, Phys. Lett. B 454, 187 (1999).

[25] T. R. Govindarajan, V. Suneeta and S. Vaidya, Nucl. Phys. B 583, 291 (2000).

[26] D. Birmingham, K. S. Gupta and S. Sen, Phys. Lett. B 505, 191 (2001).

[27] V. Moretti and N. Pinamonti, Nucl. Phys. B 647, 131 (2002).

[28] A. Jevicki and J. P. Rodrigues, Phys. Lett. B 146, 55 (1984).

[29] A. K. Das and S. A. Pernice, Nucl. Phys. B 561, 357 (1999).

[30] A. K. Das, "Supersymmetry in singular quantum mechanics," arXiv:hep-th/0005042 (2000).

[31] - F. Atiyah, V. K. Patodi, and I. M. Singer. Math. Proc. Camb. Phil. Soc., $77: 43,1975$.

[32] Handbook of Mathematical Functions. M. Abramowitz and I. Stegun editors. Dover Publications, New York (1970).

[33] Mathematica 4. Wolfram Research, Inc., Champaign, USA (1999).

A) IflP, Departamento de Física - Facultad de Ciencias Exactas, UnlP, C.C. 67 (1900) La Plata, Argentina

E-MAIL: FALOMIR@FISICA.UNLP.EDU.AR - FAX: (54 221) 425-2006

B) Departamento de Matemática - Facultad de Ciencias Exactas, UnlP, C.C. 172 (1900) La Plata, Argentina

E-MAIL: MARIAM@MATE.UNLP.EDU.AR

C) Iflp, Departamento de Física - Facultad de Ciencias Exactas, UnlP, C.C. 67 (1900) La Plata, Argentina

E-MAIL:PISANI@FISICA.UNLP.EDU.AR

D) University of Massachusetts at Boston, Boston, MA 02125, USA E-MAIL: RTS@MATH.UMB.EDU, R-SEELEY@ATTBI.COM 